\title{
Mapping the tidally disrupting Andromeda XXVII and its stellar stream
}

\author{
Janet Preston ${ }^{1}$, Michelle Collins ${ }^{2}$, Ana Bonaca ${ }^{3}$, Rodrigo Ibata $^{4}$, Erik \\ Tollerud $^{5}$, Marla Geha ${ }^{3}$ and the PAndAS collaboration \\ ${ }^{1}$ Department of Physics, University of Surrey, Guildford, GU2 7XH, Surrey, UK. \\ email: j.preston@surrey.ac.uk \\ ${ }^{2}$ Department of Physics, University of Surrey, Guildford, GU2 7XH, Surrey, UK. \\ ${ }^{3}$ Astronomy Department, Yale University, New Haven, CT 06520, USA \\ ${ }^{4}$ Observatoire de Strasbourg, 11, rue de l'Université, F-67000, Strasbourg \\ ${ }^{4}$ Space Telescope Science Institute, Baltimore, MD 21218, U.S.A
}

\begin{abstract}
Andromeda XXVII is a dwarf spheroidal galaxy in the outskirts of the Andromeda galaxy (M31). It appears to be dissolving in to the Northern arc of M31, and could be the remnant of a strong tidal disruption. In the upcoming months, our spectroscopic program, which has measured velocities for multiple stars within both the dwarf galaxy progenitor and its stream (using the Keck II DEIMOS telescope, as part of the PAndAS survey), will determine velocity dispersions, scale radii and metallicities of both the dwarf and the stream. This in turn may enable us to ascertain the progenitor mass profile and determine whether it is cusped or cored.
\end{abstract}

Keywords. galaxies: dwarf, kinematics and dynamics

\section{Current Knowledge}

The discovery of Andromeda XXVII (And XXVII) was announced by Richardson et al. in 2011, at which time it was noted that: "And XXVII appears to be in the process of being tidally disrupted by M31 and is embedded in a great northern arc of stars which loops around M31".

That same year, Carlberg et al. also affirmed that: "The association of globular clusters in the NW stream indicates that a dwarf galaxy, likely And XXVII, is the source of the stream".

This view was reinforced by Collins et al. who, in 2013, published a further analysis of And XXVII which: confirmed that the stars lie within an area of the CMD that would indicate they belong to an older ( 10 Gyr) stellar population; presented new results on the kinematics; and concluded that And XXVII was tidally disrupting and may no longer be a gravitationally bound system.

\section{Future Analysis}

New data has been obtained from the Keck II DEIMOS telescope of stars in the And XXVII dwarf galaxy and stellar stream during 2015 (see Figure1(a) for on-sky locations of the masks). This will be used in the upcoming project to:

- Confirm that stars within the new data are members of the galaxy and its stream.

- Determine velocity distributions, scale radii and metallicities of the dSph and its stream. 


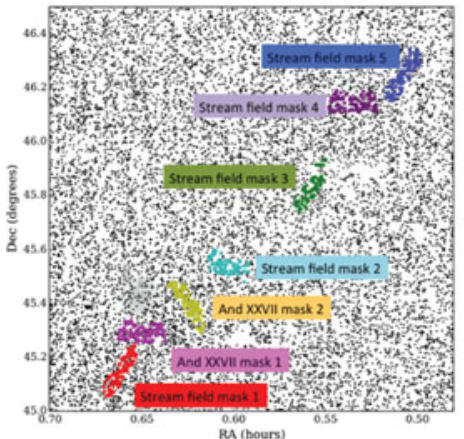

(a)

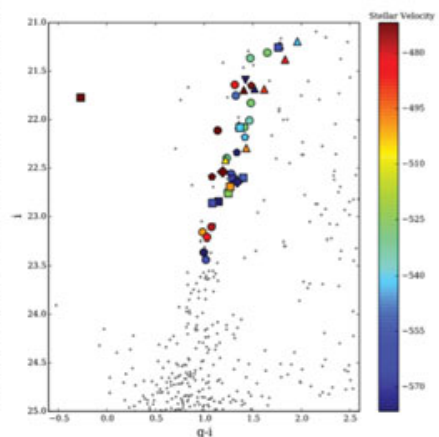

(b)



(c)

Figure 1.

(a) On-sky positions of the data obtained from objects in And XXVII and its stellar stream.

(b) Preliminary findings for the stars in the study (represented by the shapes, shaded by their systemic, heliocentric, velocities) all except one of which appear to lie on the RGB. The smaller dots represent PAndAS photometry for stars located within 3 arcmins of the centre of And XXVII.

(c) Velocity histograms for stars within And XXVII and its stellar stream for each mask. The systemic velocity of the dSph is plotted using a solid black line and is flanked by dotted lines indicating the upper and lower limits.

- Ascertain the shape of And XXVII's mass profile and use methods devised by Errani et al. (2015) to determine whether And XXVII is cusped or cored.

\section{Early Findings}

Preliminary work done to date appears to indicate; that stars within And XXVII and its stellar stream have colour magnitude profiles comparable with previous findings; and that their velocities are consistent with the systemic velocity of $-539.6_{-4.5}^{+4.7} \mathrm{kms}^{-1}$ determined by Collins et al. (2013), (see Figures 1 (b) and (c)) which is quite distinct from the systemic velocity of the M31 halo $\left(\sim-300 \mathrm{~km}^{-1}\right)$ reported by Chapman et al. (2006).

\section{References}

Carlberg, R. G., Richer, H. B., McConnachie, A. W., Irwin, M., Ibata, R. A., Dotter, A. L., Chapman, S., Fardal, M., Ferguson, A. M. N., Lewis, G. F., Navarro, J. F., Puzia, T. H., \& Valls-Gabaud, D. 2011, ApJ, 731:124

Chapman, S. C., Ibata, R., Lewis, G. F., Ferguson, A. M. N., Irwin, M., McConnachie, A., \& Tanvir, N. 2006, ApJ, 653:255

Collins, M. L. M., Chapman, S. C., Rich, R. M., Ibata, R. A., Martin, N. F., Irwin, M. J., Bate, N. F., Lewis, G. F., Pearrubia, J., Arimoto, N., Casey C. M., Ferguson, A. M. N., Koch, A., McConnachie, A. W., \& Tanvir, N. 2013, ApJ, 768:172

Errani, R., Pearrubia, J., \& Tormen, G. 2015, arXiv,1501.04968v2 [astro-ph.GA]

Richardson, J. C., Irwin, M. J., McConnachie, A. W., Martin, N. F., Dotter, A. L.,Ferguson, A. M. N., Ibata, R. A., Chapman, S. C., Lewis, G. F., Tanvir, N. R., \& Rich, R. M. 2011, ApJ, $732: 76$ 